\title{
Surgery on Herman rings of the complex standard family
}

\author{
NÚRIA FAGELLA $\dagger$ and LUKAS GEYER $\ddagger$ \\ $\dagger$ Universitat de Barcelona, Departamento de Matemàtica Aplicada i Anàlisi, \\ Gran Via 585, Barcelona, Spain \\ (e-mail: fagella@maia.ub.es) \\ $\ddagger$ Universität Dortmund, Fachbereich Mathematik Vogelpothsweg 87, \\ D-44221 Dortmund, Germany \\ (e-mail:geyer@math.uni-dortmund.de)
}

(Received 3 July 2001 and accepted in revised form 17 September 2002)

\begin{abstract}
We consider the standard family (or Arnold family) of circle maps given by $f_{\alpha, \beta}(x)=x+\alpha+\beta \sin (x)(\bmod 2 \pi)$, for $x, \alpha \in[0,2 \pi), \beta \in(0,1)$ and its complexification $F_{\alpha, \beta}(z)=z e^{i \alpha} \exp \left[\frac{1}{2} \beta\left(z-\frac{1}{z}\right)\right]$. If $f_{\alpha, \beta}$ is analytically linearizable, there is a Herman ring around the unit circle in the dynamical plane of $F_{\alpha, \beta}$. Given an irrational rotation number $\theta$, the parameters $(\alpha, \beta)$ such that $f_{\alpha, \beta}$ has rotation number $\theta$ form a curve $T_{\theta}$ in the parameter plane. Using quasi-conformal surgery of the simplest type, we show that if $\theta$ is a Brjuno number, the curve $T_{\theta}$ can be parametrized real-analytically by the modulus of the Herman ring, from $\beta=0$ up to a point $\left(\alpha_{0}, \beta_{0}\right)$ with $\beta_{0} \leq 1$, for which the Herman ring collapses. Using a result of Herman and a construction in I. N. Baker and P. Domínguez (Complex Variables 37 (1998), 67-98) we show that for a certain set of angles $\theta \in \mathcal{B} \backslash \mathcal{H}$, the point $\beta_{0}$ is strictly less than 1 and, moreover, the boundary of the Herman rings with the corresponding rotation number have two connected components which are quasi-circles, and do not contain any critical point. For rotation numbers of constant type, the boundary consists of two quasi-circles, each containing one of the two critical points of $F_{\alpha, \beta}$.
\end{abstract}

\section{Introduction}

The standard family of maps of the circle is a two-parameter family given by

$$
f_{\alpha, \beta}(x)=x+\alpha+\beta \sin (x) \quad(\bmod 2 \pi),
$$

for $x, \alpha \in[0,2 \pi)$ and $\beta \in(0,1)$. These maps are simple perturbations of rigid rotations and it is well understood how their dynamics vary in terms of the parameters $\alpha$ and $\beta$. 


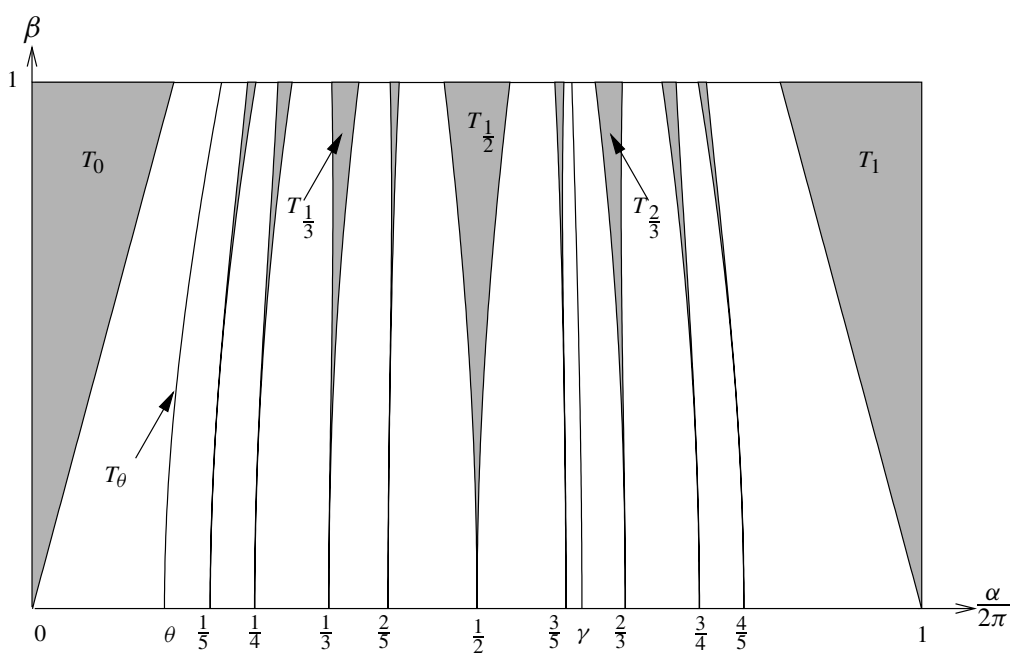

FIGURE 1. Rational Arnold tongues in the parameter space of the standard family up to denominator 5. Irrational tongues for $\gamma=(\sqrt{5}-1) / 2$ and $\theta=\sqrt[5]{2}-1$.

We define the rotation number of $f_{\alpha, \beta}$ as

$$
\theta\left(f_{\alpha, \beta}\right)=\frac{1}{2 \pi} \lim _{n \rightarrow \infty} \frac{f_{\alpha, \beta}^{n}(x)-x}{n}
$$

where $x$ is any point in the unit circle $\mathbb{T}=\mathbb{R} / 2 \pi \mathbb{Z}$. In fact, the limit on the right-hand side is independent of the point $x$ and measures the asymptotic rate of rotation of $f_{\alpha, \beta}$. With our normalization, the rotation number is rational if and only if $f$ has a periodic orbit.

The level sets $T_{\theta}$ of the rotation number $\theta$ in the parameter plane have been successfully studied. These level sets are called the Arnold tongues (see Figure 1). They are curves connecting $\{\beta=0\}$ with $\{\beta=1\}$ if the rotation number is irrational and they have an interior if it is rational. We shall be interested in the irrational case.

Maps of the standard family are real-analytic. As a consequence, if the rotation number $\theta$ of a given map $f_{\alpha, \beta}$ is irrational, $f_{\alpha, \beta}$ is topologically conjugate to the rigid rotation $r_{\theta}(x)=x+2 \pi \theta(\bmod 2 \pi)$; i.e. there exists a homeomorphism $\varphi: \mathbb{T} \rightarrow \mathbb{T}$ such that $f_{\alpha, \beta}=\varphi \circ r_{\theta} \circ \varphi^{-1}$. The regularity of $\varphi$ (in the general case of an analytic circle diffeomorphism $f$ ) has been studied for a long time. If $\varphi$ is analytic, we say that $f_{\alpha, \beta}$ is analytically linearizable. Arnold showed in [Ar] that this is the case if we require the rotation number $\rho\left(f_{\alpha, \beta}\right)=\theta$ to satisfy a certain Diophantine condition and additionally suppose that $f_{\alpha, \beta}$ is close to the rigid rotation. This result was later improved by Rüssmann, Herman and Yoccoz.

The sharpest results are due to Yoccoz [Y]. His Global Conjugacy Theorem states that if $\theta \in \mathcal{H}$, then any analytic circle diffeomorphism with rotation number $\theta$ is analytically linearizable. The set $\mathcal{H}$ of irrational numbers has full Lebesgue measure and includes all Diophantine numbers. It is strictly included in the set of Brjuno numbers $\mathcal{B}$. (See $[\mathbf{P M}]$ for precise definitions of these sets.) This result is sharp in the following sense: if $\theta \notin \mathcal{H}$, there exists an analytic circle diffeomorphism $f$ with rotation number $\theta$, such that $f$ is not analytically linearizable. Yoccoz's local conjugacy theorem states that if $f$ has rotation 
number $\theta \in \mathcal{B}$ and is close to the rigid rotation, then it is analytically linearizable. For the standard family, closeness to the rigid rotation translates into having $0<\beta<M(\theta)$, where $M(\theta)>0$ is possibly very small. For the standard family, the local conjugacy theorem is sharp. Indeed, it was shown by Geyer $[\mathbf{G}]$ that if $f_{\alpha, \beta}$ is analytically linearizable, then $\theta\left(f_{\alpha, \beta}\right) \in \mathcal{B}$.

Hence, from the point of view of analytic linearizability, there are three different types of irrational curves in the parameter space of the standard family:

(1) if $\theta \in \mathcal{H}$, all maps in $T_{\theta}$ are analytically linearizable;

(2) if $\theta \in \mathcal{B} \backslash \mathcal{H}$ and $(\alpha, \beta) \in T_{\theta}$ then there exists $M(\theta) \in(0,1]$ such that $f_{\alpha, \beta}$ is analytically linearizable for $0<\beta<M(\theta)$;

(3) if $\theta \notin \mathcal{B}$, no map in $T_{\theta}$ is analytically linearizable.

Observe that in the second case, nothing is said about those maps in $T_{\theta}$ with $\beta>M(\theta)$. In this paper, we aim to study irrational curves of type 1 and 2. More precisely, given an irrational curve $T_{\theta}$ such that $\theta \in \mathcal{B}$, we shall investigate the set of parameters $(\alpha, \beta) \in T_{\theta}$ such that $f_{\alpha, \beta}$ is analytically linearizable. In the case $\theta \in \mathcal{H}$, this set is the whole curve $T_{\theta}$ but if $\theta \in \mathcal{B} \backslash \mathcal{H}$, it might, a priori, be a disconnected set.

We are also interested in the regularity of the curves $T_{\theta}$. It follows from results in Arnold [Ar] and Herman [Her1] that $T_{\theta}$ is real-analytic if $\theta$ satisfies some Diophantine condition. Using different techniques, Risler $[\mathbf{R}]$ proved that if $\theta \in \mathcal{B}$ then $T_{\theta}$ is realanalytic for $0<\beta<N(\theta)$ with some constant $N(\theta)>0$. These results apply to more general families of analytic circle diffeomorphisms.

We shall use a complex analytic approach in order to investigate these questions. Observe that the standard family can be extended to the complex plane where it takes the form $F_{\alpha, \beta}(z)=e^{i \alpha} z \exp \left(\frac{1}{2} \beta\left(z-\frac{1}{z}\right)\right)$. This is a family of holomorphic selfmaps of $\mathbb{C}^{*}$, with 0 and $\infty$ as essential singularities. Maps of this type are studied in [Ke1, Ko1, Ko2, B, BD, Mak] among others. The dynamics of $F_{\alpha, \beta}$ and the complex parameter space of the standard family are investigated in [F1] and [F2]. Note that $F_{\alpha, \beta}$ is symmetric with respect to the unit circle $\mathbb{T}$ which is invariant; i.e. $\tau \circ F_{\alpha, \beta}=F_{\alpha, \beta} \circ \tau$ where $\tau(z)=1 / \bar{z}$. (Note: we use $\mathbb{T}$ both for $\mathbb{R} / 2 \pi \mathbb{Z}$ and for $\{|z|=1\} \subset \mathbb{C}$. The meaning should always be clear from the context.)

The dynamical plane of a holomorphic map $F$ consists of two totally invariant components: the Fatou set $\mathcal{F}(F)$, where the dynamics is stable and the Julia set $\mathcal{J}(F)=\overline{\mathbb{C}} \backslash \mathcal{F}(F)$ where chaotic dynamics occurs. The possible connected components of the Fatou set are completely classified for maps with a finite number of singular values (singularities of $F^{-1}$ which may be critical points or asymptotic values) (see [MSS, EL, GK, B, Ke1, Ko2]). Among these components we find Herman rings. An invariant Herman $\operatorname{ring} U$ of $F$ is a maximal invariant domain conformally equivalent to a round annulus $A_{R}=\{z \in \mathbb{C} \mid$ $1 / R<|z|<R\}$ for some $R>1$, on which the dynamics is holomorphically conjugate to an irrational rigid rotation $R_{\theta}(z)=e^{2 \pi i \theta} z$ on $A_{R}$; i.e. $f_{\left.\right|_{U}}=\varphi \circ R_{\theta} \circ \varphi^{-1}$ where $\theta \in \mathbb{R} \backslash \mathbb{Q}$ and $\varphi: A_{R} \rightarrow U$ is conformal. The number $R$ is unique and the modulus of $U$ is $\bmod \left(A_{R}\right)=\pi^{-1} \log R$.

From this point of view, the question of $f_{\alpha, \beta}$ being analytically linearizable is equivalent to asking whether $F_{\alpha, \beta}$ has an invariant Herman ring around the unit circle (see Figure 2), since any analytic conjugacy $\varphi$ of $\mathbb{T}$ can be extended to a conformal conjugacy on a 


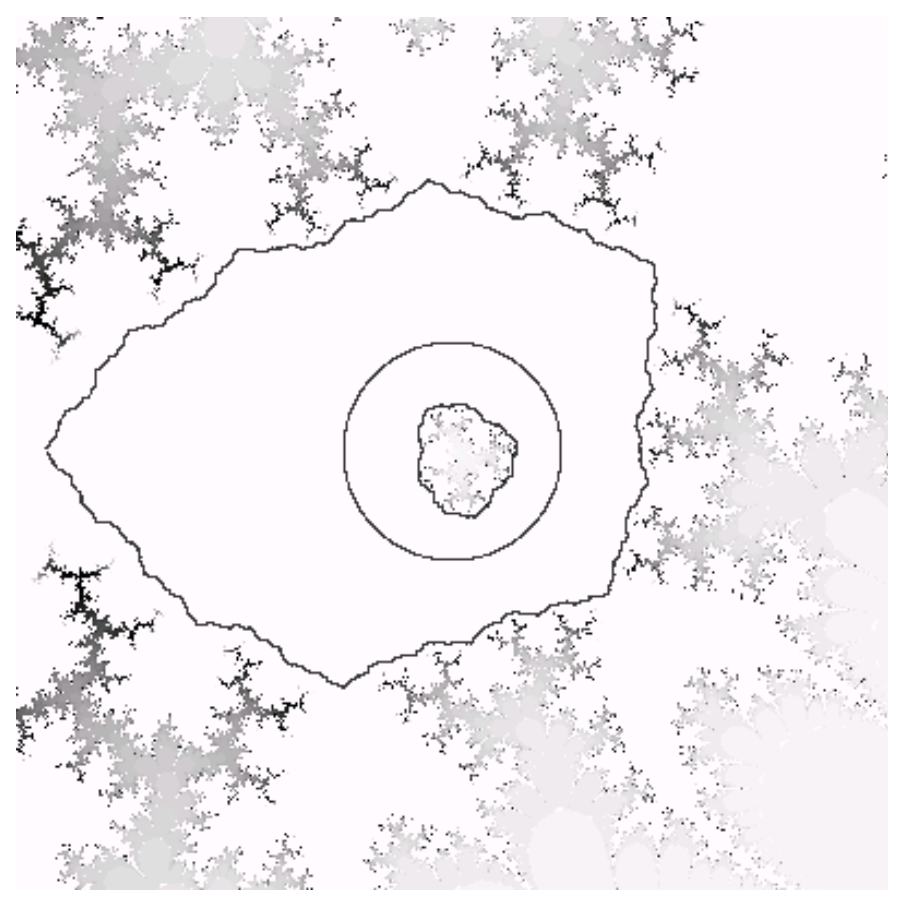

FIGURE 2. Numerical plot of the dynamical plane of $F_{\alpha, \beta}$, for $\alpha=1.9$ and $\beta=0.5$. Points in white are in the Fatou set. The orbits of the two critical points and the orbit of 1 (the unit circle) have been plotted.

neighbourhood of $\mathbb{T}$, i.e. an annulus. In this case, the Fatou set of $F_{\alpha, \beta}$ consists exclusively of the Herman ring and its pre-images (see $[\mathbf{F 2}]$ and $[\mathbf{G}])$. If $f_{\alpha, \beta}$ has irrational rotation number but is not analytically linearizable, it was shown in $[\mathbf{G}]$ that the Julia set of $F_{\alpha, \beta}$ must be the whole plane.

We know that if $\theta \in \mathcal{B} \backslash \mathcal{H}$, maps in $T_{\theta}$ with $\beta$ small enough have a Herman ring. As we increase $\beta$ on the curve $T_{\theta}$, does this ring collapse at some point before arriving at $\beta=1$ ? Or, in an extreme case, does the ring appear and disappear intermittently? We shall see that the latter situation never occurs.

Our main result in this paper is the following theorem.

Theorem A. Let $(\alpha, \beta) \in T_{\theta}$ be such that $F_{\alpha, \beta}$ has a Herman ring $U$ around $\mathbb{T}$ (of rotation number $\theta$ ). Let $R \in(1, \infty)$ be such that $\bmod (U)=\pi^{-1} \log R$. Then there exists a real-analytic map

$$
\begin{aligned}
\gamma:(0, \infty) & \longrightarrow T_{\theta} \\
t & \mapsto(\alpha(t), \beta(t))
\end{aligned}
$$

such that:

(1) for each $t \in(0, \infty)$, the map $F_{\alpha(t), \beta(t)}$ has a Herman ring $U_{t}$ of modulus $\pi^{-1} t \log R$;

(2) the map $t \mapsto \beta(t)$ is strictly decreasing;

(3) $\lim _{t \rightarrow \infty} \beta(t)=0$;

(4) $\lim _{t \rightarrow 0} \beta(t)=\beta_{0} \leq 1$; and

(5) for any $(\alpha, \beta) \in T_{\theta}$ such that $\beta \geq \beta_{0}$, the map $F_{\alpha, \beta}$ has no Herman ring. 
From this theorem we conclude that the irrational tongues $T_{\theta}$ are analytically parametrized by the modulus of the Herman ring up to a certain point $\left(\alpha_{0}, \beta_{0}\right)$ where the ring collapses. To our knowledge, this does not follow from the general regularity results mentioned earlier. After the point $\beta_{0}$, the ring never appears again. If $\theta \in \mathcal{H}$, then it follows from Yoccoz' global linearization theorem that $\beta_{0}=1$. The obvious question at this point is whether the case $\beta_{0}<1$ ever occurs and if so, for which rotation numbers $\theta$.

Using a result in [Her2], combined with a construction in [BD] and some ideas from $[\mathbf{G}]$, we give a positive answer to this question and additionally obtain information about the geometry of the Herman rings. (This surgical construction is independently carried out in [Hen].) The precise statement is as follows.

THEOREM B. For any $\beta_{0} \in(0,1)$, there exists $\alpha_{0} \in(0,2 \pi)$ such that $\left(\alpha_{0}, \beta_{0}\right) \in T_{\theta}$ for some $\theta \in \mathcal{B} \backslash \mathcal{H}$ and $F_{\alpha_{0}, \beta_{0}}$ has no Herman ring. Furthermore, if $(\alpha, \beta) \in T_{\theta}$ and $\beta<\beta_{0}$, the boundary of the Herman ring $U$ of $F_{\alpha, \beta}$ consists of two quasi-circles not containing the critical points of $F_{\alpha, \beta}$.

The geometric properties of Herman rings and the regularity properties of their boundaries are of independent interest. The following result shows that 'nice' rotation numbers induce nice boundaries.

THEOREM C. Let $\theta$ be an irrational number of constant type and let $(\alpha, \beta) \in T_{\theta}$. Let $U$ be the Herman ring of $F_{\alpha, \beta}$. Then $\partial U$ consists of two quasi-circles, each one containing a critical point of $F_{\alpha, \beta}$.

Results similar to Theorems A and C have been proved independently by Henriksen [Hen] for a family of Blaschke fractions.

There are still many open questions and directions for further research. Recently, Petersen and Zakeri used 'trans-quasi-conformal surgery' to prove that for almost all rotation numbers the Siegel discs of the quadratic family are Jordan domains containing the critical point in the boundary [PZ]. If one could transfer their techniques to the Arnold family, Theorem $\mathrm{C}$ could be considerably strengthened. Whether it is possible to have a Herman ring whose closure contains 0 and $\infty$ is still an open question about the geometry of Herman rings. Another interesting open question is whether the Arnold family is a 'prototype' for the global linearization theorem, i.e. whether $\beta_{0}<1$ for all rotation numbers $\theta \in \mathcal{B} \backslash \mathcal{H}$. There is, as yet, no explicit prototype family which is known to have this property.

\section{Quasi-conformal surgery and conformal structures}

The main tool in all proofs is quasi-conformal surgery, so we shortly recall the relevant definitions and tools. The standard references for quasi-conformal mappings are [Ah] and $[\mathbf{L V}]$.

By an almost complex structure $\sigma$ of maximal dilatation $K$ on some open set $U$, we mean a measurable field of ellipses in the tangent space of $U$, centered at 0 and defined up to multiplication with a non-zero real constant, with the ratio of major and minor axes bounded by $K$. Equivalently, one can think of $\sigma$ as represented by a measurable function $\mu: U \rightarrow \mathbb{C}$ with $|\mu(z)| \leq(K-1) /(K+1)<1$ almost everywhere. The precise relation is 
that $\arg \mu(z)$ is twice the angle between the major axis of the ellipse and the imaginary axis, and $|\mu(z)|=(K(z)-1) /(K(z)+1)$ where $K(z)$ is the ratio of the major and minor axes of the ellipse at $z$. Two such structures are equivalent if they coincide almost everywhere in $U$.

A homeomorphism $f: U \rightarrow V$ is $K$-quasi-conformal if it has locally integrable weak derivatives and satisfies

$$
\left|f_{\bar{z}}\right| \leq \frac{K-1}{K+1}\left|f_{z}\right| \text { almost everywhere. }
$$

A map $f$ is $K$-quasi-regular if it is the local composition $g \circ h$ of a holomorphic map $g$ and a $K$-quasi-conformal map $h$. The pull-back of an almost complex structure $\sigma$ defined on $V$ by a non-constant quasi-regular map $f: U \rightarrow V$ is defined as the ellipse field $f^{*} \sigma$ obtained by pulling back the ellipses by the tangent map at every point where $f$ is differentiable with a non-zero derivative. (We also say that we transport $\sigma$ by $f$.) In terms of $\mu$, this is

$$
f^{*} \mu:=\frac{f_{\bar{z}}+(\mu \circ f) \overline{f_{z}}}{f_{z}+(\mu \circ f) \overline{f_{\bar{z}}}} .
$$

Note that pulling back by holomorphic functions does not increase the maximal dilatation. The standard complex structure corresponding to $\mu_{0} \equiv 0$ is a field of circles. By integration of an almost complex structure we mean the construction of a quasiconformal map $f$ on $U$ with $f^{*} \sigma_{0}=\sigma$ (respectively $\mu=f_{\bar{z}} / f_{z}$ ), almost everywhere.

The well-known measurable Riemann mapping theorem developed by Morrey, Ahlfors (see $[\mathbf{A h}]$ ), Bers and Bojarski states that every almost complex structure is integrable. Furthermore, if $\sigma_{t}$ is an analytic family of conformal structures on $\mathbb{C}$, the integrating quasiconformal maps $\varphi_{t}$, normalized by fixing 0 and 1 , depend analytically on $t$.

\section{Proof of Theorem A}

Throughout this section, we fix $\theta \in \mathcal{B}$. Let $(\alpha, \beta) \in T_{\theta}$ be such that $F_{\alpha, \beta}$ has a Herman ring $U$ around $\mathbb{T}$ of rotation number $\theta$. We know that this ring must be invariant and the only doubly-connected component of the Fatou set $[\mathbf{B}]$. Since $F_{\alpha, \beta}$ and $\tau$ commute, it follows that $U$ is symmetric with respect to $\mathbb{T}$. Let $R \in(1, \infty)$ be such that $\bmod (U)=$ $\pi^{-1} \log R$.

The proof follows ideas from [Man] and the main tool is quasi-conformal surgery of the simplest type, i.e. a change in the complex structure.

For each $t \in(0, \infty)$ we shall put a new invariant almost complex structure $\sigma_{t}$ on $U$, and transport it to the whole complex plane by $F_{\alpha, \beta}$. After integrating $\sigma_{t}$ we will obtain a new map $G_{t}$ which is quasi-conformally conjugate to $F_{\alpha, \beta}$ and whose Herman ring $\widetilde{U}_{t}$ has modulus $\pi^{-1} t \log R$ and rotation number $\theta$ (see Figure 3 ). We finally show that the map $G_{t}$ (or an affine conjugate of it) is a member of the standard family $F_{\alpha(t), \beta(t)}$ and study the properties of the map $t \mapsto(\alpha(t), \beta(t))$.

We now proceed to make these ideas precise. Let $\varphi: A_{R} \longrightarrow U$ be the unique conformal map such that $\varphi^{-1}(1) \in \mathbb{R}^{+}$which maps the exterior boundary component of $A_{R}$ onto the exterior boundary component of $U$. Then $\varphi$ conjugates $F_{\alpha, \beta}$ on $U$ to the rigid rotation $R_{\theta}$ on $A_{R}$, i.e.

$$
\varphi \circ R_{\theta}=F_{\alpha, \beta} \circ \varphi .
$$



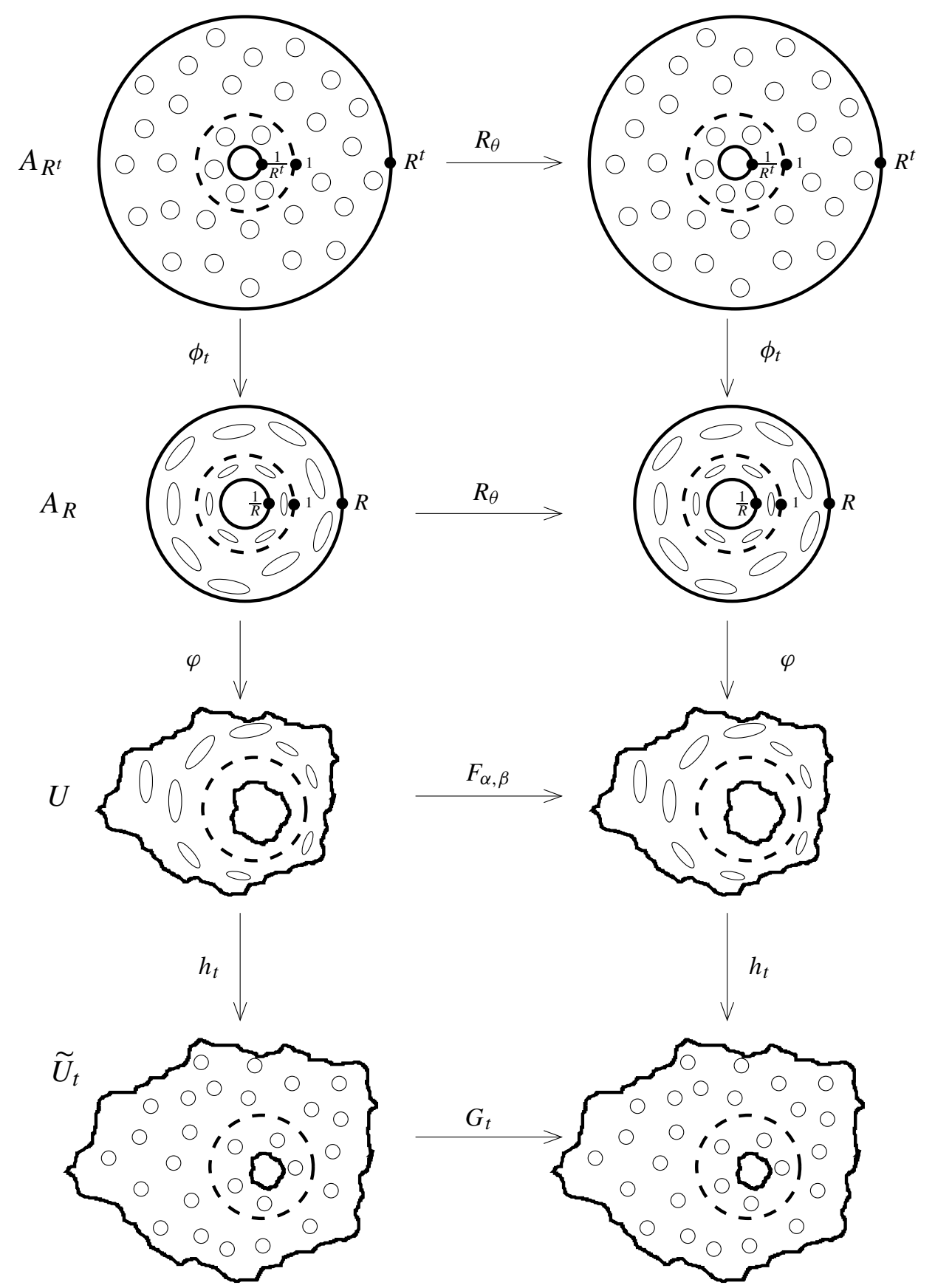

FIGURE 3. Commutative diagram summarizing the proof of Theorem A. 
LEMMA 3.1. $\varphi$ is symmetric with respect to $\mathbb{T}$, i.e. $\varphi \circ \tau=\tau \circ \varphi$. Hence $\mathbb{T}$ is $\varphi$-invariant and $\varphi(1)=1$.

Proof. Define $\tilde{\varphi}:=\tau \circ \varphi \circ \tau$. Both $A_{R}$ and $U$ are symmetric with respect to $\mathbb{T}$, so this is again a conformal map from $A_{R}$ to $U$, satisfying $\tilde{\varphi}^{-1}(1)=\tau\left(\varphi^{-1}(1)\right) \in \tau\left(\mathbb{R}^{+}\right)=\mathbb{R}^{+}$. Furthermore, it maps the exterior boundary component of $A_{R}$ onto the exterior boundary component of $U$. Since a conformal map of an annulus with this normalization is unique, we obtain $\varphi=\tilde{\varphi}$.

For $t \in(0, \infty)$, let $\phi_{t}: A_{R^{t}} \longrightarrow A_{R}$ be the quasi-conformal homeomorphism $\phi_{t}(z)=z|z|^{t^{-1}-1}$. This map commutes with $R_{\theta}$ and satisfies $\phi_{t}(1)=1$. Clearly, $\mathbb{T}$ is invariant under $\phi_{t}$ and $\tau \circ \phi_{t}=\phi_{t} \circ \tau$.

Let $\sigma_{0}$ be the standard complex structure. We define a new complex structure $\sigma_{t}$ in $\mathbb{C}^{*}$ as follows:

$$
\sigma_{t}= \begin{cases}\left(\left(\varphi \phi_{t}\right)^{-1}\right)^{*}\left(\sigma_{0}\right) & \text { on } U, \\ \left(F_{\alpha, \beta}^{n}\right)^{*}\left(\sigma_{t}\right) & \text { on } F_{\alpha, \beta}^{-n}(U) \text { for all } n \geq 1, \\ \sigma_{0} & \text { on } \mathbb{C}^{*} \backslash \bigcup F_{\alpha, \beta}^{-n}(U) .\end{cases}
$$

We observe that $\sigma_{t}$ has a bounded distortion for any $t \in \mathbb{R}^{+}$and it is invariant by $F_{\alpha, \beta}$ since $\varphi \circ \phi_{t}$ conjugates $F_{\alpha, \beta}$ to $R_{\theta}$.

We proceed to integrate this complex structure. By the Measurable Riemann Mapping Theorem, there exists a unique quasi-conformal homeomorphism $h_{t}: \mathbb{C} \longrightarrow \mathbb{C}$ fixing 0 and 1 and such that $\left(h_{t}\right)^{*}\left(\sigma_{0}\right)=\sigma_{t}$.

We define $G_{t}:=h_{t} \circ F_{\alpha, \beta} \circ h_{t}^{-1}$. By construction, $G_{t}: \mathbb{C}^{*} \rightarrow \mathbb{C}^{*}$ is holomorphic and is conjugate to $F_{\alpha, \beta}$.

Moreover, the map $\psi_{t}:=h_{t} \circ \varphi \circ \phi_{t}: A_{R^{t}} \longrightarrow h_{t}(U)$ is conformal, and it conjugates $G_{t}$ on $h_{t}(U)$ to the rigid rotation $R_{\theta}$ on $A_{R^{t}}$. Hence, $\widetilde{U}_{t}:=h_{t}(U)$ is a Herman ring of rotation number $\theta$ for the map $G_{t}$. Clearly, $\bmod \left(\widetilde{U}_{t}\right)=\pi^{-1} \log R^{t}=\pi^{-1} t \log R$.

Lemma 3.2. The map $G_{t}$ is symmetric with respect to $\mathbb{T}$.

Proof. By construction, $\sigma_{t}$ is symmetric with respect to $\mathbb{T}$, hence necessarily the integrating map is, too. It follows (similarly as in Lemma 3.1) that $G_{t}$ is also symmetric with respect to $\mathbb{T}$.

We now want to show that the map $G_{t}$ (or an affine conjugate of it) is a member of the standard family. This will follow from the next proposition which we state in a more general form.

Proposition 3.1. Let $G: \mathbb{C}^{*} \rightarrow \mathbb{C}^{*}$ be a holomorphic map with essential singularities at 0 and $\infty$. Suppose that $G$ is symmetric with respect to the unit circle (i.e. $G \tau=\tau G$ ). Assume, moreover, that $G$ has exactly two simple critical points none of which is on $\mathbb{T}$. Then,

$$
G(z)=z e^{i \alpha} \exp \left(B\left(z-\frac{\bar{B}}{B z}\right)\right)
$$

where $\alpha \in(0,2 \pi)$ and $B \in \mathbb{C}^{*}$. 
Proof. The map $G$ is a holomorphic map of $\mathbb{C}^{*}$ of finite type, hence

$$
G(z)=z^{n} A \exp (P(z)-Q(1 / z)),
$$

where $P$ and $Q$ are polynomials with no constant term, of degrees $d_{p}, d_{q} \geq 1$ [Ke2]. Since $G$ is a homeomorphism of $\mathbb{T}$ and $n$ is the winding number of the image curve of $\mathbb{T}$, we must have $n=1$.

First we impose that $G$ has only two critical points. The derivative is

$$
G^{\prime}(z)=A e^{P(z)-Q(1 / z)}\left(1+z P^{\prime}(z)+\frac{Q^{\prime}(1 / z)}{z}\right) .
$$

Hence, the critical points are the solutions of the following equation:

$$
z^{2} P^{\prime}(z)+z+Q^{\prime}(1 / z)=0 .
$$

Now, we know that

$$
Q^{\prime}(1 / z)=\frac{\widetilde{Q}(z)}{z^{d_{q}-1}},
$$

where $\widetilde{Q}$ is a polynomial of degree $d_{q}-1$ with a non-zero constant term. Hence equation (1) can be written as

$$
z^{d_{q}+1} P^{\prime}(z)+z^{d_{q}}+\widetilde{Q}(z)=0 .
$$

Since there are only two critical points, this equation must have degree two. Since $d_{q} \geq 1$, we have that $d_{q}=1$ and $P^{\prime}(z)$ must be a constant. Therefore, $d_{p}=1$ and

$$
G(z)=z A \exp \left(B\left(z-\frac{C}{z}\right)\right)
$$

for some $A, B, C \in \mathbb{C}^{*}$.

Writing the symmetry with respect to $\mathbb{T}$ explicitly, we obtain

$$
|A|^{2} \exp \left(\frac{1}{\bar{z}}\left(\bar{z}^{2}(\bar{B}-B C)+B-\overline{B C}\right)\right) \equiv 1
$$

This implies $|A|=1$ and $\bar{B}-B C=0$, thus

$$
G(z)=z e^{i \alpha} \exp \left(B\left(z-\frac{\bar{B}}{B z}\right)\right)
$$

for some $\alpha \in \mathbb{R} / 2 \pi \mathbb{Z}$, and some $B \in \mathbb{C}^{*}$.

We return to the proof of Theorem A. It follows from Proposition 3.1 that the map $G_{t}$ is of the form

$$
G_{t}(z)=z e^{i \alpha(t)} \exp \left(B(t)\left(z-\frac{\overline{B(t)}}{B(t) z}\right)\right)
$$

where $\alpha(t) \in \mathbb{R} / 2 \pi \mathbb{Z}$ and $B(t) \in \mathbb{C}^{*}$.

Observe that, if $B(t) \in \mathbb{R}$, the map $G_{t}$ is a member of the standard family. But, with the conditions we have imposed, this is not necessarily true. The critical points of $G_{t}$ are symmetric with respect to $\mathbb{T}$ but they are not necessarily on the negative real line. The next 
step is to compose $G_{t}$ with a rigid rotation that moves the critical points to the negative real line or, equivalently, that makes $B(t)$ real.

Let

$$
\rho_{t}(w):=\frac{|B(t)|}{B(t)} w
$$

and define

$$
\tilde{F}_{t}(w):=\rho_{t}^{-1} \circ G_{t} \circ \rho_{t}(w)=w e^{i \alpha(t)} \exp \left(|B(t)|\left(w-\frac{1}{w}\right)\right) .
$$

This map is an element of the Arnold family with $\beta(t):=2|B(t)|$.

The map $\tilde{F}_{t}=F_{\alpha(t), \beta(t)}$ has $U_{t}=\rho_{t}^{-1}\left(h_{t}(U)\right)$ as a Herman ring with rotation number $\theta$ and modulus $\pi^{-1} t \log R$. This proves the existence of the map $\gamma$ and the first statement of Theorem A. We proceed now with the proof of statements (2)-(5).

Proposition 3.2. The map $t \longmapsto(\alpha(t), \beta(t))$ is real-analytic.

Proof. We will first show that $t \longmapsto B(t)$ is real-analytic, where $B(t)$ is as before (i.e. the complex parameter of $\left.G_{t}\right)$. The real-analyticity of $\beta(t)=2|B(t)|$ is an easy consequence because $B(t) \neq 0$. Let $\widetilde{c}(t)$ denote the critical point of $G_{t}$ inside the unit disc; i.e. one of the solutions of

$$
B(t) z^{2}+z+\bar{B}(t)=0 .
$$

This equation must be equal to

$$
A(t)(z-\widetilde{c}(t))\left(z-\frac{\widetilde{c}(t)}{|\widetilde{c}(t)|^{2}}\right)=0
$$

for some constant $A(t)$ that makes the coefficient of $z$ equal to 1 . After some easy calculations, one can write it as

$$
\frac{-|\widetilde{c}(t)|^{2}}{\widetilde{c}(t)\left(1+|\widetilde{c}(t)|^{2}\right)} z^{2}+z-\frac{\tilde{c}}{\left(1+|\widetilde{c}(t)|^{2}\right)}=0
$$

and hence

$$
B(t)=\frac{-|\widetilde{c}(t)|^{2}}{\widetilde{c}(t)\left(1+|\widetilde{c}(t)|^{2}\right)} .
$$

Now, if we let $c$ denote the critical point of $F_{\alpha, \beta}$ inside the unit disc and since the maps $F_{\alpha, \beta}$ and $G_{t}$ are conjugate, we have that, for all $t$,

$$
\widetilde{c}(t)=h_{t}(c)
$$

Hence,

$$
B(t)=\frac{-\left|h_{t}(c)\right|^{2}}{h_{t}(c)\left(1+\left|h_{t}(c)\right|^{2}\right)} .
$$

By the Measurable Riemann Mapping Theorem with dependence on parameters, we know that the map $t \mapsto h_{t}(c)$ is real-analytic. Hence $t \mapsto B(t)$ is also real-analytic in the domain of definition.

The real-analyticity of $t \mapsto \alpha(t)$ follows from a similar argument. If we let $v=F_{\alpha, \beta}(c)$ be the critical value of $F_{\alpha, \beta}$ inside the unit disc, then $\tilde{v}(t)=G_{t}(\tilde{c}(t))=h_{t}(v)$ is the critical 
value of $G_{t}$ in the unit disc. It moves real-analytically with $t$, again by the Measurable Riemann Mapping Theorem. Furthermore,

$$
e^{i \alpha(t)}=\tilde{v}(t) \tilde{c}(t)^{-1} \exp \left(-B(t)\left(\tilde{c}(t)-\frac{\overline{B(t)}}{B(t) \tilde{c}(t)}\right)\right) .
$$

This shows that the left-hand side and thus $\alpha(t)$ moves real-analytically, too.

LEMMA 3.3. The map $t \longmapsto \beta(t)$ is injective.

Proof. Suppose $\beta\left(t_{1}\right)=\beta\left(t_{2}\right)$. Since both $F_{\alpha\left(t_{1}\right), \beta\left(t_{1}\right)}$ and $F_{\alpha\left(t_{2}\right), \beta\left(t_{2}\right)}$ have a Herman ring of rotation number $\theta$, we have that both pairs of parameters belong to the irrational curve $T_{\theta}$.

But it is well known that the irrational curves $T_{\theta}$ are graphs in the $\alpha-\beta$ plane, i.e. there are no two different points in any irrational curve with the same parameter $\beta$ (see, e.g., [MS, Lemma 4.1, Ch. I]). Hence $\alpha\left(t_{1}\right)=\alpha\left(t_{2}\right)$. As the Herman rings of $F_{\alpha\left(t_{1}\right), \beta\left(t_{1}\right)}$ and $F_{\alpha\left(t_{2}\right), \beta\left(t_{2}\right)}$ have modulus $\pi^{-1} t_{1} \log R$ and $\pi^{-1} t_{2} \log R$, respectively, we get $t_{1}=t_{2}$.

The following lemma ensures that all maps in the curve $T_{\theta}$ with parameter $\beta$ less than the original one have a Herman ring.

LeMMA 3.4. As $t$ tends to $\infty$, the parameter $\beta(t)$ tends to 0 .

For the proof we shall need the following theorem on conformal mapping of large annuli.

THEOREM 3.1. [Man, Mc] Let $R_{n}$ be a sequence of positive numbers that tends to $\infty$ as $n$ tends to $\infty$. Let $\psi_{n}: A_{R_{n}} \rightarrow \mathbb{C}^{*}$ be a sequence of univalent maps with $\psi_{n}(1)=1$ and with the image of $\psi_{n}$ separating 0 and $\infty$. Then, $\psi_{n}$ converges to the identity on compact sets of $\mathbb{C}^{*}$.

Proof of Lemma 3.4. Consider the sequence of maps

$$
\psi_{t}:=h_{t} \circ \varphi \circ \phi_{t}: A_{t} \longrightarrow h_{t}(U),
$$

which are all normalized by $\psi_{t}(1)=1$, by construction. By Theorem 3.1, this sequence converges uniformly to the identity on compact sets. Hence, for any $M>0$ and any $\varepsilon>0$, we can find $t_{0}$ such that for all $t>t_{0}$,

$$
\left|\psi_{t}(z)-z\right|<\varepsilon \quad \text { for all } z \in A_{M} .
$$

This means that, for any $M>0$, and for $t$ large enough, the Herman ring $\widetilde{U}(t)=h_{t}(U)$ contains the round annulus $A_{M}$. Hence, the Herman ring $U_{t}=\rho_{t}^{-1}(\widetilde{U}(t))$ also contains $A_{M}$ for $t$ large enough. Therefore,

$$
\frac{1}{M}<c(t)<0
$$

where $c(t)=\rho_{t}^{-1}\left(h_{t}(c)\right)$ is the critical point of $F_{\alpha(t), \beta(t)}$ inside the unit disc. Now, as $t$ tends to infinity, we can make $1 / M$ tend to 0 and hence $c(t)$ tends to 0 .

By a computation similar to that of $B(t)$ one can see that

$$
\beta(t)=\frac{2 c(t)}{-1-c(t)^{2}},
$$

and therefore $\beta(t)$ tends to 0 as $t$ tends to infinity. 
It follows from this fact and from the injectivity of $\beta(t)$ (see Lemma 3.3) that $\beta(t)$ is strictly decreasing with $t$. It remains only to see what happens when $t$ tends to 0 . Since no Herman ring may exist for any $\beta \geq 1$ (because at this point $F_{\alpha, \beta}$ ceases to be a homeomorphism), and $\beta(t)$ is a continuous function, we must have

$$
\lim _{t \rightarrow 0} \beta(t)=\beta_{0} \leq 1
$$

The final point is as follows.

LEMMA 3.5. If $\beta_{0}<1$, and $\left(\alpha^{\prime}, \beta^{\prime}\right) \in T_{\theta}$ are such that $\beta^{\prime} \geq \beta_{0}$, then $F_{\alpha^{\prime}, \beta^{\prime}}$ has no Herman ring.

Proof. If there exists $\beta^{\prime} \geq \beta_{0}$ and $F=F_{\alpha^{\prime}, \beta^{\prime}} \in T_{\theta}$ with a Herman ring, then the previous procedure applied to $F$ shows that there is a non-zero lower bound on the modulus of the Herman ring of $F_{\alpha(t), \beta(t)}$ for any $t$, contradicting the fact that these moduli tend to zero as $t$ tends to zero.

This concludes the proof of Theorem A.

\section{Proof of Theorem B}

An essential ingredient is the following unpublished result of Michel Herman on general families of circle diffeomorphisms.

THEOREM 4.1. [Her2] Let $f$ be an orientation-preserving $\mathcal{C}^{\infty}$-diffeomorphism of $\mathbb{R} / 2 \pi \mathbb{Z}$ such that no iterate of $f+\alpha$ lifts to the identity map, for any $\alpha \in \mathbb{R}$. Then, there exists $\alpha \in \mathbb{R}$ such that:

(1) $f+\alpha$ has an irrational rotation number $\theta$;

(2) $f+\alpha=\varphi \circ r_{\theta} \circ \varphi^{-1}$ where $\varphi$ is a quasi-symmetric map of $\mathbb{T}$ and $\varphi(1)=1$; and

(3) $\varphi$ is not $\mathcal{C}^{2}$.

For any $\beta_{0} \in(0,1)$ let $\alpha_{0}$ be given by Theorem 4.1, taking $f=f_{0, \beta_{0}}$. Then $f_{\alpha_{0}, \beta_{0}}$ is a diffeomorphism of the circle which is quasi-symmetrically conjugate to the rigid rotation $r_{\theta}$ but not $\mathcal{C}^{2}$ conjugate. In particular, $F_{\alpha_{0}, \beta_{0}}$ has no Herman ring.

In what follows we will construct a one-parameter curve of maps of the standard family $F_{\alpha(s), \beta(s)}$ for $s \in(1, \infty)$, each of them having a Herman $\operatorname{ring} U_{s}$ of rotation number $\theta$ and modulus $\pi^{-1} \log s$. Observe that, by Theorem A, $s \mapsto(\alpha(s), \beta(s))$ is a reparametrization of $T_{\theta}$ up to $\beta_{0}$ and hence it covers all maps in $T_{\theta}$ with a Herman ring. The boundaries of the rings $U_{s}$ we construct will be quasi-circles not containing the critical points of $F_{\alpha(s), \beta(s)}$.

The idea of the construction is as follows.

We start in the dynamical plane of $F_{\alpha_{0}, \beta_{0}}$. We make some space around $\mathbb{T}$ in order to glue a round annulus $A_{s}$ there. Then we define a new self-map of $\mathbb{C}^{*}, H_{s}$, which equals the rigid rotation $R_{\theta}$ inside $A_{s}$ and equals $F_{\alpha_{0}, \beta_{0}}$ (after a change of scale) outside this annulus. This map is not holomorphic. We construct an $H_{s}$-invariant almost complex structure $\sigma_{s}$ on $\mathbb{C}^{*}$ to obtain, after applying the measurable Riemann mapping theorem, a holomorphic map $G_{S}$ with the desired dynamical properties.

We now proceed to make this construction precise. From now on, let $F=F_{\alpha_{0}, \beta_{0}}$ to simplify the notation. 
Fix any $s \in(1, \infty)$ and consider the round annulus $A_{s}=\{1 / s<|z|<s\}$. Let $\varphi: \mathbb{T} \rightarrow \mathbb{T}$ be the quasi-symmetric map that conjugates $f_{\alpha_{0}, \beta_{0}}$ to the rigid rotation $r_{\theta}$.

Define

$$
H_{s}^{(1)}(z)= \begin{cases}s F(z / s) & \text { for }|z| \geq s \\ s^{-1} F(s z) & \text { for }|z| \leq s^{-1}\end{cases}
$$

and observe the symmetry $\tau \circ H_{s}^{(1)}=H_{s}^{(1)} \circ \tau$. Note that the previous dynamics of $F$ on $\mathbb{T}$ now occur under $H_{s}^{(1)}$ on each of the components of the boundary of $A_{s}$ which we denote by $C_{1 / s}$ (inner) and $C_{s}$ (outer). More precisely, it follows that $\varphi(z s)$ (respectively $\varphi(z / s)$ ) conjugates quasi-symmetrically $H_{s}^{(1)}$ on $C_{1 / s}$ (respectively $C_{s}$ ) to $R_{\theta}$ on $\mathbb{T}$.

In order to extend the mapping on the boundary to a mapping on the whole annulus we need the following standard extension lemma.

LemMa 4.1. For $s>1$, let $C^{(i)}$ and $C^{(o)}$ denote respectively the inner and outer boundary of the round annulus $A_{s}=\{1 / s<|z|<s\}$. Let $\varphi^{(i)}: C^{(i)} \rightarrow C^{(i)}$ and $\varphi^{(o)}: C^{(o)} \rightarrow C^{(o)}$ be two quasi-symmetric maps symmetric with respect to $\mathbb{T}$, i.e. satisfying $\tau \circ \varphi^{(i)}=\varphi^{(o)} \circ \tau$. Then there exists a quasi-conformal map $\varphi: A_{s} \rightarrow A_{S}$ such that:

(1) $\varphi_{C_{C^{(i)}}}=\varphi^{(i)}$ and $\varphi_{C_{C^{(o)}}}=\varphi^{(o)}$; and

(2) $\varphi$ is symmetric with respect to $\mathbb{T}$ i.e. $\tau \circ \varphi=\varphi \circ \tau$.

One proves this lemma by first extending the maps by Beurling-Ahlfors or DouadyEarle extension to some small neighbourhood of the boundary curves, so we have a situation where the boundary maps are real-analytic, though the moduli of the annuli might be different. One proceeds by applying the extension lemmas from, e.g., [G] or [Kr]. The main idea there is to lift everything by universal coverings to parallel strips, where one just uses linear interpolation. The whole construction can be made in a symmetric way, so the resulting map will be symmetric with respect to the unit circle.

We now apply Lemma 4.1 and obtain a quasi-conformal map $\varphi_{s}: A_{s} \rightarrow A_{s}$, symmetric with respect to $\mathbb{T}$ and satisfying

$$
\varphi_{s}(z)= \begin{cases}s \varphi(z / s) & \text { for } z \in C_{s} \\ s^{-1} \varphi(z s) & \text { for } z \in C_{1 / s}\end{cases}
$$

which is the map we shall use to glue the rigid rotation into $A_{s}$. Indeed, we finally define the new map $H_{S}$ to be

$$
H_{s}= \begin{cases}H_{s}^{(1)} & \text { on } \mathbb{C} \backslash A_{s} \\ \varphi_{s}^{-1} \circ R_{\theta} \circ \varphi_{s} & \text { on } A_{s} .\end{cases}
$$

It is easy to check that the two parts match on the boundaries of $A_{S}$ and thus $H_{S}$ is a continuous map of $\mathbb{C}^{*}$. Inside $A_{s}$ it is quasi-conformal, outside it is analytic, so $H_{S}$ is quasi-regular in $\mathbb{C}^{*}$. Moreover, $H_{S}$ is symmetric with respect to $\mathbb{T}$ and has exactly two critical points bounded away from $A_{s}$, since the critical points of $F$ were bounded away from $\mathbb{T}$. 
We now have a map with the desired dynamics. We proceed to construct an invariant almost complex structure with bounded dilatation ratio, to obtain a holomorphic map conjugate to $H_{s}$. We start by setting the standard complex structure $\sigma_{0}$ on $A_{s}$, transported to $A_{s}$ by the quasi-conformal map $\varphi_{s}$. We then transport this structure to the whole plane using the map $H_{s}$. More precisely, define

$$
\sigma_{s}= \begin{cases}\left(\varphi_{s}\right)^{*} \sigma_{0} & \text { on } A_{s} \\ \left(H_{s}^{n}\right)^{*} \sigma_{s} & \text { on } H_{s}^{-n}\left(A_{s}\right) \text { for all } n \geq 1 \\ \sigma_{0} & \text { on } \mathbb{C}^{*} \backslash \bigcup_{n=1}^{\infty} H_{s}^{-n}\left(A_{s}\right) .\end{cases}
$$

By construction, $\sigma_{s}$ is invariant under $H_{s}$ and has a bounded distortion, since $\varphi_{s}$ is quasiconformal and $H_{s}$ is holomorphic outside $A_{s}$. Moreover, $\sigma_{s}$ is symmetric with respect to $\mathbb{T}$.

Applying the measurable Riemann mapping theorem we obtain a unique quasiconformal homeomorphism $h_{s}: \mathbb{C} \rightarrow \mathbb{C}$ which fixes 0 and 1 and integrates $\sigma_{s}$. Hence, the map $G_{s}:=h_{s} \circ H_{s} \circ h_{s}^{-1}$ is a holomorphic map of $\mathbb{C}^{*}$ with essential singularities at 0 and $\infty$. It is easy to check that $G_{s}$ has a Herman ring which is exactly $h_{s}\left(A_{s}\right)$. Indeed, $G_{s}$ is conformally conjugate to the rigid rotation on this annulus. The conformal conjugacy is given by $\varphi_{s} \circ h_{s}^{-1}$. Moreover, if the rotation domain of $G_{s}$ extended beyond $h_{s}\left(A_{s}\right)$ then we could follow the construction backwards and use symmetry to conclude that the original map $F$ would have a Herman ring around the unit circle, contradicting the fact that $\varphi$ is not real-analytic.

By construction, $G_{s}$ is symmetric with respect to the unit circle. Moreover, it is clear that $G_{s}$ has only two critical points which are not on the unit circle and, in fact, they are bounded away from the boundary of the Herman ring, since the critical points of the original map $F$ were bounded away from the unit circle. Hence, $G_{s}$ satisfies all hypotheses in Proposition 3.1. It follows that the map can be written as

$$
G_{s}(z)=z e^{i \alpha(s)} \exp \left(B(s)\left(z-\frac{\overline{B(s)}}{B(s) z}\right)\right)
$$

where $\alpha(s) \in \mathbb{R} / 2 \pi \mathbb{Z}$ and $B(s) \in \mathbb{C}^{*}$. As in the proof of Theorem A, we conjugate $G_{s}$ by the rotation

$$
\rho_{s}(w)=\frac{|B(s)|}{B(s)} w
$$

and obtain the new map $\rho_{s}^{-1} \circ G_{s} \circ \rho_{s}=F_{\alpha(s), \beta(s)}$ which is a member of the standard family conjugate to $G_{s}$. The set $U_{s}=\rho_{s}^{-1}\left(h_{s}\left(A_{s}\right)\right)$ is a Herman ring of rotation number $\theta$ and modulus $\pi^{-1} \log s$, as required.

\section{Proof of Theorem $C$}

This theorem follows essentially from the results of $[\mathbf{G}]$, combined with Theorem A. Here is a sketch of the proof. We know from [G] that the map $E_{\theta}(z)=e^{2 \pi i \theta} z e^{z}$ has a Siegel disc which is a quasidisc with a critical point on the boundary, because $\theta$ is of constant type. We now choose some invariant curve $\gamma$ inside the Siegel disc and paste the dynamics of $E_{\theta}$ outside $\gamma$ with the dynamics of itself, conjugated by reflection with respect 
to $\gamma$, inside $\gamma$. The resulting map is an element of the Arnold family with rotation number $\theta$ which has a Herman ring bounded by two quasi-circles containing the critical points. (This construction is detailed in the aforementioned paper.) Now by Theorem A any two elements of the Arnold family which have a Herman ring of the same rotation number are quasi-conformally conjugate. The properties of being a quasi-circle and containing a critical point are invariant under quasi-conformal conjugation, so Theorem $\mathrm{C}$ is proved.

Acknowledgements. We wish to thank C. Petersen, A. Douady and X. Buff for helpful discussions. Many thanks to C. Henriksen and to the referee for carefully reading the paper and providing valuable suggestions.

NF was partially supported by DGICYT Grant No PB96-1153, BFM2000-0805-C02-01 and CIRIT 2001SGR-70.

\section{REFERENCES}

[Ah] L. Ahlfors. Lectures on Quasiconformal Mappings. Wadsworth and Brooks/Cole Mathematics Series, 1966.

[Ar] V. I. Arnold. Small denominators I: on the mappings of a circle into itself. Translations of the Amer. Math. Soc., 2nd series 46 (1961), 213-284.

[B] I. N. Baker. Wandering domains for maps of the punctured plane. Ann. Acad. Sci. Fenn., Ser. A. I. Math. 12 (1987), 191-198.

[BD] I. N. Baker and P. Domínguez. Analytic self-maps of the punctured plane. Complex Variables 37 (1998), 67-98.

[EL] A. Eremenko and M. Lyubich. Dynamical properties of some classes of entire functions. Ann. Inst. Fourier 42 (1992), 989-1020.

[F1] N. Fagella. Limiting dynamics of the complex standard family. Int. J. Bif. Chaos 3 (1995), 673-700.

[F2] N. Fagella. Dynamics of the complex standard family. J. Math. Anal. Appl. 229 (1999), 1-31.

[G] L. Geyer. Siegel discs, Herman rings and the Arnold family. Trans. Amer. Math. Soc. 353(9) (2001), 3661-3683.

[GK] L. Goldberg and L. Keen. A finiteness theorem for a dynamical class of entire functions. Ergod. Th. \& Dynam. Sys. 6 (1986), 183-192.

[Hen] C. Henriksen. Holomorphic dynamics and Herman rings. Master's Thesis, Technical University of Denmark, 1997.

[Her1] M. Herman. Sur la conjugaison différentiable des difféomorphismes du cercle à des rotations. Inst. Hautes Études Sci. Publ. Math. 49 (1979), 5-233.

[Her2] M. Herman. Conjugaison quasi-symmétrique des difféomorphismes du cercle à des rotations et applications aux disques singuliers de Siegel I. Unpublished manuscript.

[Ke1] L. Keen. Dynamics of holomorphic self-maps of $\mathbb{C}^{*}$. Proc. Workshop on Holomorphic Functions and Moduli. Springer, Berlin, 1988, pp. 9-30.

[Ke2] L. Keen. Topology and growth of a special class of holomorphic self-maps of $\mathbb{C}^{*}$. Ergod. Th. \& Dynam. Sys. 9 (1989), 321-328.

[Ko1] J. Kotus. Iterated holomorphic maps of the punctured plane. Dynamical Systems (Lecture Notes Economics and Mathematical Systems, 287). Eds. A. B. Kurzhanski and K. Sigmund. Springer, Berlin, 1987.

[Ko2] J. Kotus. The domains of normality of holomorphic self-maps of $\mathbb{C}^{*}$. Ann. Acad. Sci. Fenn. (Ser. A, I. Math.) 15 (1990), 329-340.

[Kr] H. Kriete. Herman's proof of the existence of critical points on the boundaries of singular domains. Progress in Holomorphic Dynamics (Pitman Research Notes, 387). Addison-Wesley, Reading, MA, 1998, pp. 31-40.

[LV] O. Lehto and K. I. Virtanen. Quasiconformal Mappings in the Plane, 2nd edn. Springer, Berlin, 1975. 
[Man] R. Mañé. On the instability of Herman rings. Invent. Math. 81 (1985), 459-471.

[Mak] P. Makienko. Iterations of analytic functions of $\mathbb{C}^{*}$ (Russian) Dokl. Akad. Nauk SSRR 297 (1987), 35-37; translation in Sov. Math. Dokl. 36 (1988), 418-420.

[MS] W. de Melo and S. van Strien. One-Dimensional Dynamics. Springer, Berlin (1993).

[Mc] C. McMullen. Complex Dynamics and Renormalization (Ann. Math. Studies, 135). Princeton University Press.

[MSS] R. Mañé, P. Sad and D. Sullivan. On the dynamics of rational maps. Ann. Sci. École Norm. Sup. 16(4) (1983), 193-217.

[PM] R. Pérez-Marco. Solution complète au problème de Siegel de linéarisation d'une application holomorphe au voisinage d'un point fixe (d'après J.-C. Yoccoz) (Séminaire Bourbaki, 1991/92). Astérisque 206 (1992), Exp. No. 753, 4, 273-310.

[PZ] C. L. Petersen and S. Zakeri. On the Julia set of a typical quadratic polynomial with a Siegel disk. IMS Preprint 00-06.

[R] E. Risler. Linéarisation des perturbations holomorphes des rotations et applications. Mém. Soc. Math. Fr. 77 (1999).

[Y] J. C. Yoccoz. Conjugaison des difféomorphismes analytiques du cercle. Preprint. 